\title{
Controlling sequence length of DS-IR-UWB to enhance performance of multi-WBAN systems
}

\author{
Nguyen Cong Dinh*, Pham Thanh Hiep*, Obinata Yua**, Vu Van Son ${ }^{*}$
}

\begin{abstract}
The international standard of wireless body area networks (WBANs), ie IEEE 802.15.6, was established in Feb. 2012, and this standard decided spreading code as a suppressing interference technology. However, the sequence length of spreading code is fixed, hence it is difficult to guarantee secure communications due to noise and interference from other WBANs. In this paper, we propose utilizing direct sequence impulse radio ultra wideband (DS-IR-UWB) for multi-WBAN systems, and then analyze multi-WBAN systems theoretically, derive equations of inter-WBAN interference, packet error rate (PER) and throughput. Furthermore, to guarantee secure communications, the desired PER is introduced, and then an algorithm is proposed to take the control of sequence length of DS-IR-UWB in order to ensure that the PER of system is always below the desired PER while maximizing the throughput. The numerical evaluation shows that the sequence length of proposed control method is changed according to the SNR and the number of WBANs, it lets the proposed algorithm of control of sequence length outperform the conventional fixed sequence length method.
\end{abstract}

K e y w o r d s: control of sequence length, Inter-WBAN interference, direct sequence impulse radio ultra wideband (DSIR-UWB), Packet error rate, throughput

\section{Introduction}

Due to rapidly increasing of elderly population all over the world and quickly developing of entertainment devices, wireless body area networks (WBANs) attract an attention to sensing vital informations for medical systems [1]. Hence, the IEEE 802.15.6 standard for WBANs was establish in Feb. 2012 due to emergency of WBANs [2].

The WBAN is a network around the human body, consists of one coordinator and several sensors in star topology. The WBAN for medical systems has two duties, sensing and controlling. Sensing is to sense vital information and controlling is to control actuator based on derived information by sensing [3]. For example, the coordinator collects the vital information from glucose sensors, and then control insulin pump. In this scenario, the WBAN is two way network and different from usual sensor networks.

Because of dealing with personal informations, reliability of WBANs should be high. However, a packet error rate (PER) is deteriorated due to additive white Gaussian noises (AWGN) and/or interferences from other WBANs that are placed in its transmission region [46]. In general cellular systems, a control of transmit power is adopted to avoid the inter-cell interference [7]. However, WBANs are different to general cellular systems, the distance from a sensor to its coordinator may longer than the distance from the sensor to adjacent coordinators. Hence, the control of transmit power is invalid for WBAN systems. The method that uses an error-correcting code to improve the reliability was proposed [8]. However, this work discussed on one WBAN scenario, this method is unsuitable for multi-WBAN scenario. The medium access control (MAC) method was also proposed for suppressing interferences from other WBANs [9]. This research controlled data delivery rate and data delivery latency while the PER is not taken into consideration. We have proposed the cluster-based topology for multi-WBAN to avoid the inter-WBAN interference (IBI) [10], and then proposed spatial reuse supperframe to enhence the throughput of cluster-based WBANs [11]. However, these methods require strict synchronization between WBANs that is complicated problem for pratical applications. In this work, we would like to proposea method which can suppress IBI without the necessity of synchronization between WBANs.

The IEEE 802. 15. 6 standard decided that one of frequencies for WBANs is an ultra wide band (UWB). It was expected for vital sensing and controlling in hospitals because of its high speed transmission and coexistent robustness with another wireless communication system [12], [13]. Thus, the UWB technology is adopted in this work. On the other hand, in IEEE802. 15. 6, the spreading code is used for suppressing interference [2], however the sequence length is fixed. Therefore, it is difficult to guarantee secure communication due to AWGN and in-

* Le Quy Don Technical Academy, Ha Noi, Viet Nam, dinhnc@mta.edu.vn, phamthanhhiep@gmail.com, sontlc246@gmail.com,** Fujitsu.co, Japan, yuao@fujitsu.jp 
terference from other WBANs. In this paper, in order to improve the performance of multi-WBAN systems without the necessity of synchronization, we propose applying a direct sequence impulse radio ultra wide band (DS-IRUWB) to WBANs. The direct sequence spread spectrum (DSSS) was proposed for multiple hops MIMO systems by the members of our research group [16]. However, due to the difference in topology of WBANs and multiple hops systems, the DSSS of multiple hops systems cannot be applied directly to WBAN systems, especially to multiWBAN systems. Therefore, in this work, we propose the application of DSSS for IR-UWB. Moreover, because the PER decreases when the sequence length of DS-IR-UWB increases, and the throughput is expected to be increased. However, the throughput decreases when the sequence length of DS-IR-UWB increases because of increasing redundancy. Consequently, we propose an algorithm to control the sequence length in order to make sure the PER of system is below the desired PER while maximazing the throughput. We represent briefly the system model of two way multi-WBAN systems, explain the control method of sequence length to suppress interferences from other WBANs based on the desired PER. Finally, the performance evaluation by numerical analysis is shown.

\section{System model}

\subsection{Concept of system}

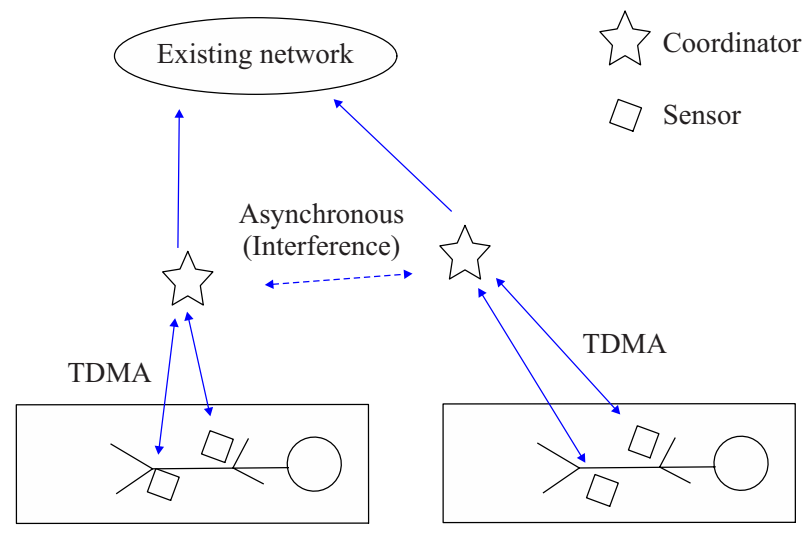

Fig. 1. Concept of multi-WBAN systems

Figure 1 shows a concept of two way multi-WBAN systems, in which there are patients reclining on beds in a hospital, and they are equipped with WBANs which consist of coordinators and sensors. Coordinators transmit data required signal to sensors in fixed intervals, and sensors reply data packets to the coordinators. Communication between sensors is unnecessary meaning the topology of WBANs is star topology. Moreover, after receiving data packets from sensors, the coordinators forward them to existing network. We assume that the transmission of coordinators and sensors is controlled by time division multiple access (TDMA) scheme, and hence the interference between sensors, between the coordinator and its own sensors is negligible. Howevre, it is difficult to synchronize multi-WBANs, therefore a WBAN is interfered by other WBANs, called inter-WBAN interference [4].

In our model, a quasi steady state is assumed to be regular whereas the other parameters, such as signal to noise ratios (SNR), the number of WBANs and so on, are changed. The propagation model is assumed to be in hospitals. Additionally, sensors are placed on the patient, consequently, the communication environment is changed slowly.

\subsection{Packet error}

We define that a packet is error when synchronizations of transmitted packet with the coordinator fails. According to IEEE 802.15.6, the transmited packet can synchronize with the coordinator by Synchronization header (SHR). SHR shall be divided into two parts, the first one is the preamble, intends for timing synchronization, packet detection, and carrier frequency offset recovery. The second one is the start-of-frame delimiter (SFD), used for frame synchronization. In IEEE 802.15.6, the size of SFD is $63 \mathrm{bits}$, and the received data packet of coordinator is error when any bit of SFD is error.

\subsection{Inter-WBAN interference (IBI)}

According to (10) in Appendix, the IBI - denoted here as $I_{B}$ - from other WBANs to a given WBAN in every chip is described by Gaussian approximation as follows

$$
I_{B}=\sum_{k=1, k \neq i}^{K} P_{k}
$$

where $P_{k}$ denotes the signal power of one chip in $k$-th WBAN and $K$ is the number of WBANs. Therefore, the signal to noise ratio $(S)$, of any WBAN can be represented by the following formula

$$
S=\frac{N m_{p} P}{N I_{B}+\sigma^{2}}
$$

where $N$ represents the sequence length of a WBAN, $\sigma^{2}$ is the power spectrum of noise and $m_{\mathrm{p}}$ is the correlation output value of spreading code. Therefore, the bit error rate (BER) of a WBAN is, [14]

$$
R^{\mathrm{bit}}=\frac{1}{2} \operatorname{erfc} \sqrt{\frac{S}{2}}
$$

The packet error rate (PER) is defined as

$$
R^{\mathrm{pac}}=1-\left[1-R^{\mathrm{bit}}\right]^{m},
$$

where $m$ is the number of bits of start-of-frame delimiter which notices the start of a data frame. 


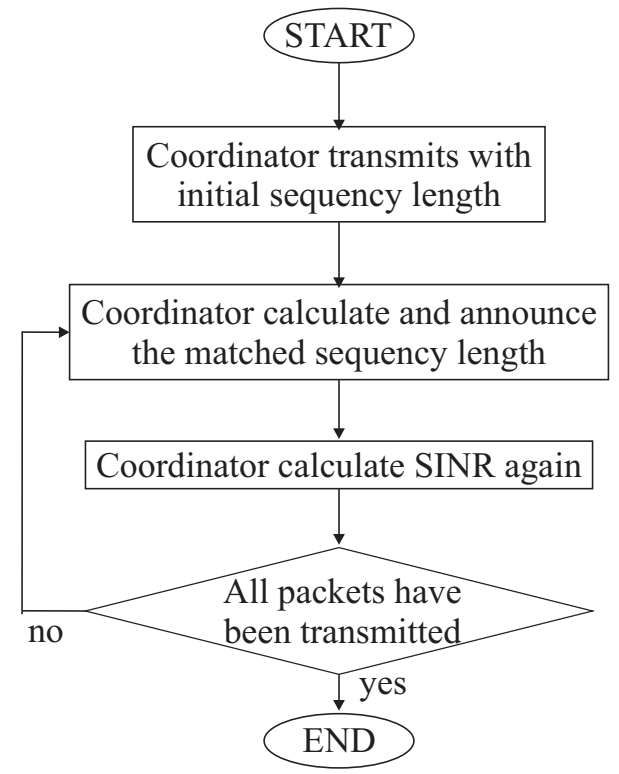

Fig. 2. The flowchart of the proposed algorithm

\subsection{Throughput}

The throughput of the WBAN is calculated as follows [15].

$$
C=\left(1-R^{\mathrm{pac}}\right) \frac{B}{N}
$$

where $B$ denotes the bandwidth.

Once the packet error rate $\left(R_{\text {pac }}\right)$ is improved, the reliability of WBANs is improved. However, in order to improve the packet error rate, see (2), the sequence length $N$ should be increased. It means that more pulses are necessary to transmit one information bit, therefore the throughput decreases.

\section{Proposed control of sequency length method}

\section{1 Algorithm}

In this section, we explain the proposed algorithm that takes the control of sequency length. The sequence length of DS-IR-UWB is adaptively changed in order to obtain the maximal throughput while the packet error rate (PER) is below its desired value. The coordinator can calculate the received signal-to-noise ratio (SNR) based on the replied packet, and then change the sequence length according to the received SNR. It is difficult for conventional multi-WBAN system to guarantee the PER rate because of fixed sequence length. Moreover, in low interference environment, the sequence length should be reduced to increase the throughput of system. Hence, in order to obtain the maximal throughput and satisfy the desired PER, our proposed control algorithm leads the sequence length match the following condition:

$$
\begin{aligned}
& \text { MAX : } \quad C_{i}=B \frac{1-R_{i}^{\text {pac }}}{N_{i}} \\
& \text { subject to } R_{i}^{\text {pac }} \leqslant \text { desired } R^{\text {pac }}
\end{aligned}
$$

The desired value $R^{\text {pac }}$ of PER is chosen according to the priority of sensors. The proposed algorithm satisfying above condition is shown in Fig. 2 and carried out as follows:

- Step 1: First, the coordinator transmits a test signal to the sensors with initial sequence length, and then calculates the SNR, based on the replied signal from the sensors.

- Step 2: According to (3) and (4), the coordinator derives the minimum sequence length that lets the PER be below the desired value.

- Step 3: The coordinator transmits a packet which includes the information of suitable sequence length to the sensors.

- Step 4: The sensors receive the packet and begin transmitting vital data packets by new sequence length.

- Step 5: The coordinator receives the vita data packet, measures SNR again.

- Step 6: If all of packets have been transmitted, this process finishes. Otherwise, return to Step 2 ocuures.

\section{Performance evaluation}

\begin{tabular}{|c|c|}
\hline Parameter & $\begin{array}{c}\text { Value } \\
\text { conventional proposal }\end{array}$ \\
\hline desired PER & 0.01 \\
\hline SFD size & 63 bits \\
\hline Center frequency & $7.9872 \mathrm{GHz}$ \\
\hline Bandwidth & $499.2 \mathrm{MHz}$ \\
\hline Modulation & direct sequence \\
\hline SNR & {$\left[\begin{array}{ll}-5 & 5\end{array}\right] \mathrm{dB}$} \\
\hline Number of WBANs & {$\left[\begin{array}{ll}1 & 10\end{array}\right]$} \\
\hline Sequence & constant \\
\hline length & $(7,15,31)$ \\
\hline
\end{tabular}

4.1 System parameters

Table 1. System parameters

Table 1 shows an example of system parameters which are used for numerical evaluation. SFD size, center frequency and bandwidth is based on IEEE 802.15.6 [2]. In this research, the is decided as 0.01 , another value of the is straightforward. Without loss of generality, the transmit power of all sensors is assumed to be the same, therefore in the worst case, the desired signal power equals to the interference signal power. 


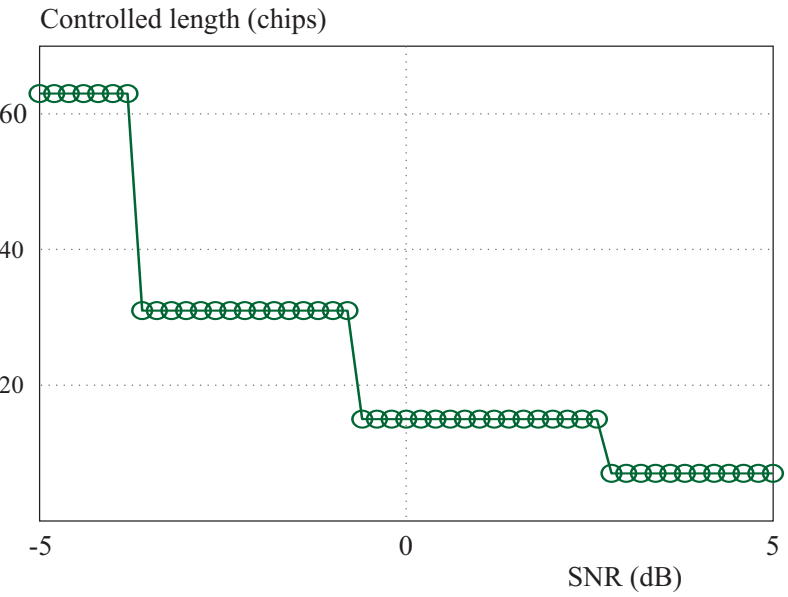

Fig. 3. The sequence length of proposed algorithm versus SNR

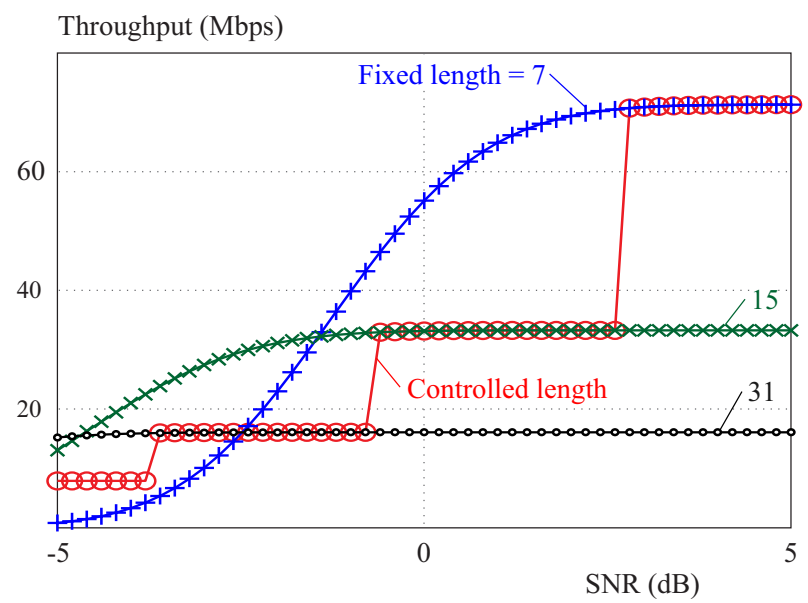

Fig. 5. Comparing the throughput of two methods

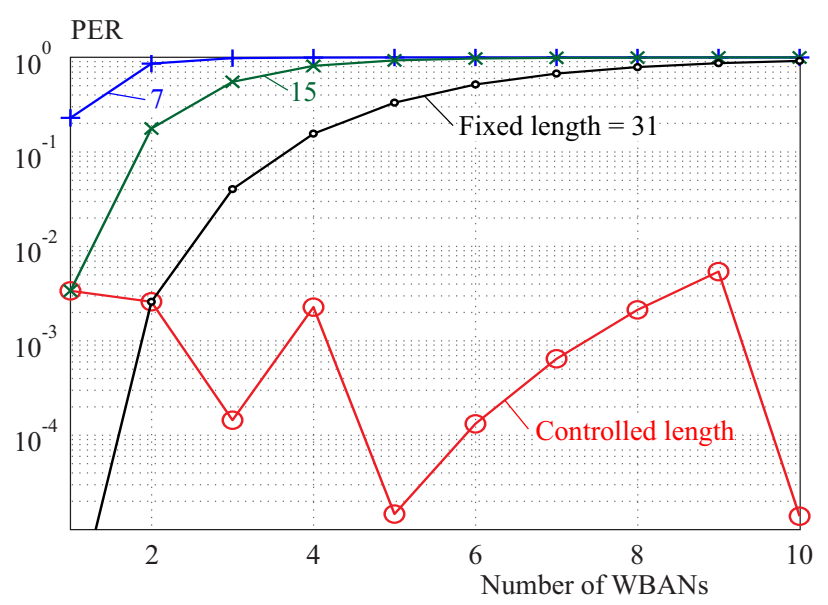

Fig. 7. Comparing the PER of two methods when the number of WBANs changes

\subsection{Numerical result}

Figures 3, 4 and 5 respectively show controlled sequence length, comparison of PER and throughput of both the conventional fixed sequence length and proposed control of sequence length under the AWGN environment.

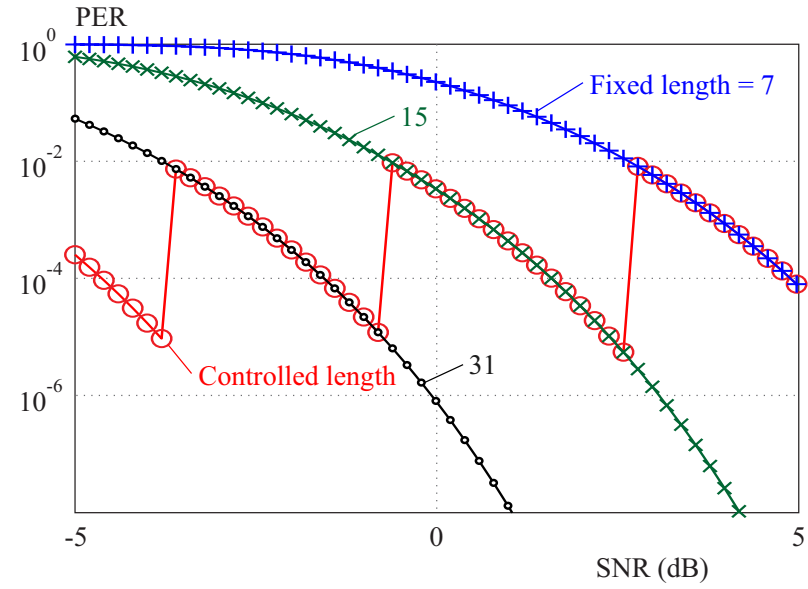

Fig. 4. The PER of fixed sequence length and controlled sequence length versus SNR

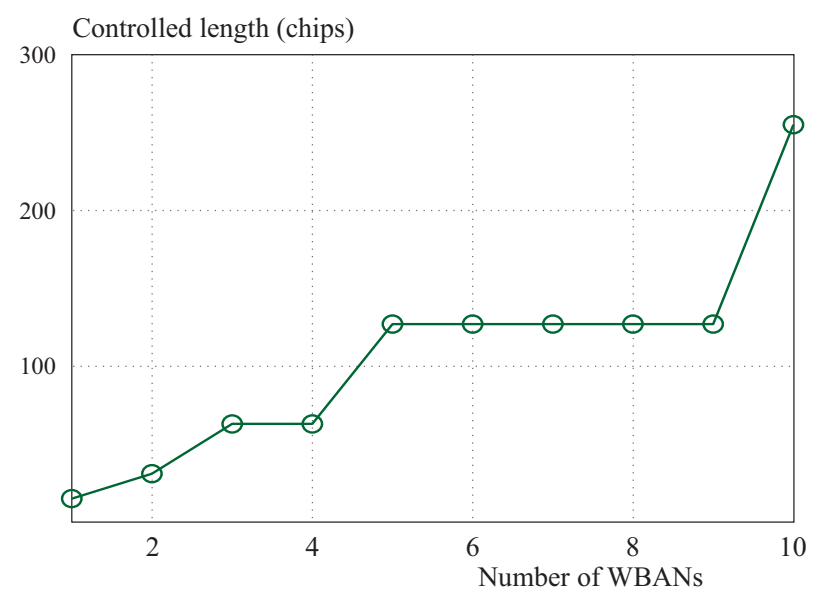

Fig. 6. The controlled sequence length of proposed method versus number of WBANs

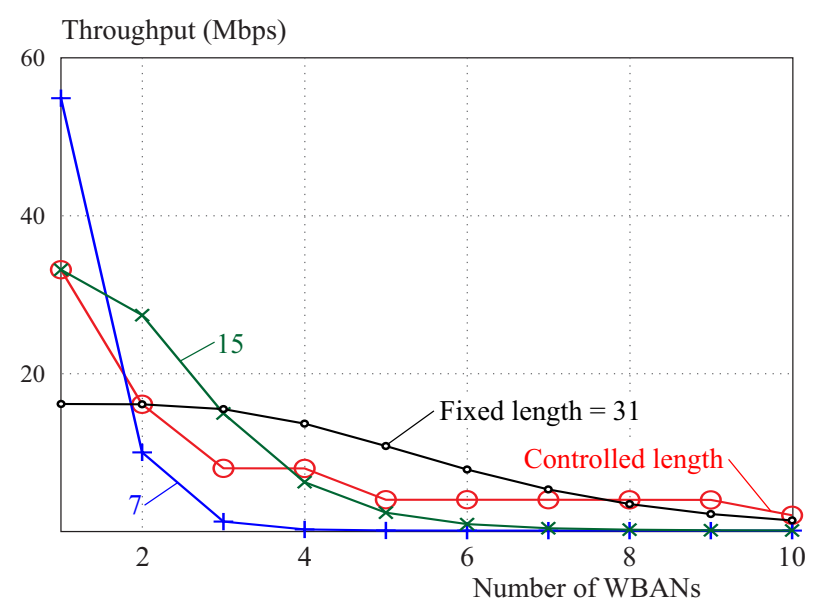

Fig. 8. The throughput of two methods versus the number of WBANs

The result in Fig. 3 shows that the sequence length of proposed method is controlled, the worse the SNR is, the longer the sequence length becomes. The sequence length is increased when the SNR decreases in order to make sure the PER is below the desired PER. As shown in Fig. 4, the PER of fixed sequence length method gets worse and 
be over the desired PER in low SNR region, whereas the PER of proposed method remains below the desired PER because of changing the sequence length according to the SNR. Additionally, the throughput of proposed method is improved while satisfying the desired PER (Fig. 5).

Similarly, Figs. 6, 7 and 8 respectively show the controlled sequence length, the comparison of PER and throughput versus the number of WBANs. The number of WBANs is changed from 1 to 10 and the SNR is fixed as $0 \mathrm{~dB}$. As shown in Fig. 6, the sequence length of proposed method becomes longer when the number of WBANs increases. The reason is, when the number of WBANs increases, the IBI also increases, therefore the sequence length should be longer in order to keep the SINR as well as the PER satisfy the conditions. It is proved in Fig. 7, the PER of proposed control of sequence length method remains below the desired PER, whereas the PER of conventional fixed sequence length method gets over the desired PER when the number of WBANs increases. Additionally, as shown in Fig. 8, the throughput of proposed method is improved while the PER is satisfying the condition, the throughput of fixed length method is lower or the PER is over the desired PER.

\section{CONCLUSION}

In this paper, we have analyzed the two way multiWBAN system using DS-IR-UWB, and derived the equations to calculate the PER and the throughput. The algorithm to control sequence length of DS-IR-UWB was proposed in order to ensure that the PER is below the derised PER while maximizing the throughput. The proposed method was compared with the conventional fixed sequence length method, and the numerical results indicated that the sequence length of proposed method is increased when the SNR decreases and/or the number of WBANs increases, it lets the PER of system satisfy above conditions, whereas the PER of conventional method comes over the desired PER or the throughput is low.

The reliability of multi-WBAN system was guaranteed by controlling sequence length of DS-IR-UWB, the other methods, such as control of transmit power, control of medium accesss and so on will be proposed in our future works.

\section{Appendix: Principle of DS-IR-UWB}

Ultra-Wideband (UWB) is used to refer to any radio technology with bandwidth exceeding lesser of $500 \mathrm{~b} \mathrm{MHz}$ or $20 \%$ of the arithmetic center frequency, according to Federal Communication Comission (FCC) [17]. A substantial change occured in February 2002, when the FCC issued that UWB could be used for data communications as well as for imaging and location applications. The band allocated to communication is from $3.1 \mathrm{GHz}$ to $10.6 \mathrm{GHz}$, a staggering $7.5 \mathrm{GHz}$. For UWB communications, there are two technologies used for system implementation, impulse radio (IR) and multiple bands [18]. The impulse radio UWB (IR-UWB) is the original approach to UWB by using narrow pulses that occupy a large portion of the spectrum. The direct spread (DS) is one of code division multiple access technologies for IR-UWB, named DS-IRUWB. The DS-IR-UWB is explained in detail as follows.

The transmit signal from the $i$-th user is expressed as, [8]

$$
s_{i}(t)=\sum_{j=-\infty}^{\infty} d_{i}(j) \sum_{l=0}^{N_{i}-1} \sqrt{P_{i}} c_{i}(l) w\left(t-j T_{s}-l T_{c}-\theta_{i}\right)
$$

where $T_{s}$ denotes the symbol time and each symbol consists of $N_{i}$ chips each of duration $T_{c}, w(t)$ represents the normalized impulse of duration $T_{w} \ll T_{c}, d_{i}(j) \in\{-1,1\}$ are the information symbols of $i$-th user and assumed to be independent and identically distributed, $\left\{c_{i}(l)\right\}_{i=0}^{N_{i}-1}$ is the multiple access code of $i$-th user, $\theta_{i}$ - is the time asynchronism that is assumed to be a uniformly distributed within $\left[0, T_{s}\right], P_{i}$ denotes the transmit power of every chip, [8].

The receiver input signal is the sum of delayed transmit signals from the different users.

Its expression is given by

$$
y(t)=\sum_{i=1}^{M} s_{i}\left(t-\tau_{i}\right)+n(t)
$$

where $n(t)$ is complex additive white gaussian noise (AWGN) with power spectrum of $\sigma^{2}$, and $M$ is the number of users. The receiver separates the signal of every user by multiplying its own spreading code, the received signal of $i$-th user is described as

$$
\begin{aligned}
& \hat{s}_{i}(t)=\int_{-\infty}^{\infty} c_{i}^{*}(t) y(t) \mathrm{d} t=N_{i} P_{i} m_{p} \sum_{j=-\infty}^{\infty} d_{i}(j)+ \\
& +\int_{-\infty}^{\infty} c_{i}^{*}(t) \sum_{l \neq i}^{M} c_{l} s_{l}(t) \mathrm{d} t+\int_{-\infty}^{\infty} c_{i}^{*}(t) n(t) \mathrm{d} t
\end{aligned}
$$

where $m_{p}$ denotes the correlation output value of spreading code. In (10), the first term is the desired signal, while the second term is the interference signals from other users and the third term is the noise.

\section{REFERENCES}

[1] H. Cao, V. Leung, C. Chow and H. Chan, "Enabling technologies for wireless body area networks: A survey and outlool,", IEEE Commun. Mag., vol.47, no.12, pp.84-93, Dec.2009.

[2] IEEE-SA Standards Board, "IEEE Standard for Local and metropolitan area networks Part 15.6: Wireless Body Area Networks,", IEEE Std 802.15.6, February 2012.

[3] B. Latre, B. Braem, I. Moerman, C. Blondia and P. Demeester, "A survey on wireless body area networks,", Wirless Network, vol.17, pp.1-18, Jan.2011. 
[4] Essafi Sarra and Tahar Ezzedine, "Performance improvement of the wireless body area network (WBAN) under interferences,", IEEE 18th International Conference on e-Health Networking, Applications and Services (Healthcom), 2016.

[5] Yong Xu, Mengya Ke, Fen Liu and Qianming Zha, "A self-adaptive Power control algorithm based on game theory for inter-WBAN interference mitigation,", 2nd IEEE International Conference on Computer and Communications (ICCC), 2016.

[6] Xiaosong Zhao, Bin Liu, Chang Chen and Chang Wen Chen, "QoS-Driven Power Control for Inter-WBAN Interference Mitigation,", IEEE Global Communications Conference (GLOBECOM), 2015.

[7] Oter. O, Pengfei. Xia, LaSita. F and Olesen. R, "Advanced power control techniques for interference mitigation in dense 802.11 networks,", IEEE International Symposium on Wireless Personal Multimedia Communications (WPMC), 2013.

[8] K. Takabayashi, abd H. Tanaka, Chika Sugimoto and R. Kohno, "Multiplexing and Error Control Scheme for Body Area Network Employing IEEE 802.15.6,", IEEE Trans. commu., vol.E97-B, No.3, 2014

[9] G.T. Chen, W.T. Chen and nd S.H. Shen, "2L-MAC: A MAC Protocol with Two-Layer Interference Mitigation in Wireless Body Area Networks for Medical Applications,", IEEE International Conference on Communications (ICC), 2014.

[10] Pham Thanh Hiep and Ryuji Kohno, "Control superframe for High Throughput of Cluster-Based WBAN with CSMA/CA", IEEE 25th International Symposium on Personal, Indoor and Mobile Radio Communications (PIMRC 2014), Washington DC, USA 2014.

[11] Pham Thanh Hiep, "Spatial Reuse Superframe for High Throughput Cluster-Based WBAN with CSMA/CA,", Ad Hoc \& Sensor Wireless Networks, Vol.31, No.1-4, pp.69-87, 2016,.
12] Atef H. Bondok, Awny M. El-Mohandes, Ahmed Shalaby and Mohammed S. Sayed, "A low complexity UWB PHY baseband transceiver for IEEE 802.15.6 WBAN,", 30th IEEE International System-on-Chip Conference (SOCC), 2017.

[13] Thanadol Tiengthong, Sathaporn Promwong and Chairak Deepunya, "Delay characteristics of UWB transmission waveform with human body for WBAN system,", International Conference on Digital Arts, Media and Technology (ICDAMT), 2018.

[14] Pham Thanh Hiep and Ryuji Kohno, "Examining exclusive transmit time for high propagation characteristic of Distributed MIMO Repeater System with amplify-and-forward,", the 8th Asia Pacific Wireless Communications Symposium (APWCS), 2011.

[15] Pham Thanh Hiep and Vu Van Son, "Joint pre-processing co-channel interference cancellation for single user MIMO,", Wireless Networks DOI 10.1007/s11276-015-1118-6, Vol.22, Iss.8, pp 25972606, 2016.

[16] Vu Van Son, Pham Thanh Hiep and Ryuji Kohno, "Spatial Reuse TDMA/CDMA in Multi-hop MIMO Relay Systems with Imperfect CSI,", Wireless Personal Communications, Vol.84, No.2, pp.1197-1208, 2015.

[17] Roberto Aiello and Anuj Batra, Ultra wideband systems: technologies and applications, Oxford, 2006.

[18] Jeff Foerster, Evan Green, Srinivasa Somayazulu and David Leeper, "Ultra-wideband technology for short or medium-range wireless communications,", Intel Techlogogy Journal, 2001.

Received 27 September 2018 